\title{
CASUÍSTICA DE ENFERMEDADES CARDIACAS EN CANINOS DE LA CLÍNICADE ANIMALES MENORES DE LA FACULTAD DE MEDICINA VETERINARIA, UNIVERSIDAD NACIONALMAYOR DE SAN MARCOS, DURANTE EL PERIODO 2007-2009
}

\section{Frequency of Heart Diseases in Dogs at the Small Animal Clinic of the Faculty of Veterinary Medicine, San Marcos University during the 2007-2009 PERIOD}

\author{
Karla Calderón O. ${ }^{1,3}$, Roberto Dávila F. ${ }^{1,4}$, César Gavidia C. ${ }^{2}$
}

\section{Resumen}

Se revisaron las fichas clínicas del total de pacientes que acudieron a la Clínica de Animales Menores de la Facultad de Medicina Veterinaria de la Universidad Nacional Mayor de San Marcos durante el periodo 2007-2009, con el fin de obtener información estadística sobre cardiopatías en caninos. De 13035 fichas clínicas se encontraron 260 canes con diagnóstico de cardiopatía. La enfermedad crónica de las válvulas auriculoventriculares fue la cardiopatía más frecuente (71.9\% de total de cardiopatías). Los machos tuvieron mayor riesgo de tener enfermedad cardiaca que las hembras $(\mathrm{OR}=1.3)$. Los grupos etarios de 9 a 12 y mayores de 12 años de edad tuvieron mayor riesgo que los menores de un año $(\mathrm{OR}=27.9$ y 75.9, respectivamente), y los canes con peso igual o menor a $10 \mathrm{~kg}$ fueron los de mayor riesgo de tener enfermedad cardiaca $(\mathrm{OR}=2.7)$. Asimismo, los Pekineses mostraron mayor predisposición a sufrir de cardiopatías (18.0\% del total de pacientes cardiópatas).

Palabras clave: frecuencia, cardiopatías, caninos, válvulas auriculoventriculares

\section{Abstract}

Clinical records from all patients attended in the Small Animal Clinic of the Faculty of Veterinary Medicine, San Marcos University during the period 2007-2009 were revised with the aim of obtaining statistical information about heart diseases in canines. Out of 13035

\footnotetext{
${ }^{1}$ Clínica de Animales Menores, ${ }^{2}$ Laboratorio de Medicina Veterinaria Preventiva, Facultad de Medicina Veterinaria, Universidad Nacional Mayor de San Marcos, Lima

${ }^{3}$ E-mail: karla_lco@hotmail.com

${ }^{4}$ E-mail: rodaf45@yahoo.com
}

Recibido: 22 de octubre de 2013

Aceptado para publicación: 12 de marzo de 2014 
clinical records, 260 cases were diagnosed with heart diseases. The chronic atrioventricular valve disease was the most frequent anomaly (71.9\% of total heart diseases). Male had a higher risk for heart disease than females $(\mathrm{OR}=1.3)$. The age groups of 9 to 12 and more than 12 years old had also higher risk than the ones younger than 1 year old $(\mathrm{OR}=27.9$ and 75.9 respectively) and dogs with equal or lower body weight of $10 \mathrm{~kg}$ had the highest risk for heart disease $(\mathrm{OR}=2.7)$. Moreover, the Pekingese breed showed greater predisposition to suffer from heart diseases (18.0\% of total heart diseases).

Key words: frequency, heart diseases, canines, atrioventricular valves

\section{INTRODUCCIÓN}

Las enfermedades cardiacas constituyen un motivo de consulta importante en la práctica clínica canina diaria, especialmente para los pacientes geriátricos y de razas predispuestas a desarrollar cardiopatías. Según Freeman y Rush (2006), las patologías cardiacas constituyen una de las principales causas de muerte en perros. En el reporte del Colegio Americano de Medicina Veterinaria Interna (Atkins et al., 2009), se estima que el $10 \%$ de perros que se presentan a consulta veterinaria básica tienen enfermedad cardiaca, y que la enfermedad crónica de las válvulas auriculoventriculares es la cardiopatía canina más común, representando cerca del $75 \%$ de casos de enfermedad cardiaca en las prácticas veterinarias de Norteamérica.

Se han realizado diversos estudios epidemiológicos con el fin de determinar la casuística de las enfermedades cardiacas en caninos. Así, se dispone de los trabajos de Detweiler et al. (1961) en Pensilvania, EEUU, Fioretti y Delli (1988) en Italia, Eichelberg y Seine (1996) en Alemania, Jourde (2002) en Francia, y Castro et al. (2009) en Brasil. Sin embargo, no se dispone en el Perú un estudio que determine la frecuencia de las enfermedades cardiacas, así como las posibles predisposiciones por efecto de la raza, sexo, edad y peso, entre otras variables.
El presente estudio tuvo como objetivo determinar la casuística de cardiopatías en perros en la Clínica de Animales Menores de la Universidad Nacional Mayor de San Marcos, la cual representa una clínica veterinaria de referencia para la ciudad de Lima.

\section{Materiales y MéTodos}

El estudio retrospectivo se llevó a cabo en la Clínica de Animales Menores (CAM) de la Facultad de Medicina Veterinaria (FMV), Universidad Nacional Mayor de San Marcos (UNMSM), en la ciudad de Lima. Se revisaron todas las historias clínicas de caninos $(n=13035)$ registradas entre 2007 a 2009.

Los criterios de inclusión fueron:

- Reporte de soplo de intensidad III/VI o superior al examen físico, asociado o no a un cuadro clínico característico, con o sin diagnóstico de cardiopatía, con o sin exámenes adicionales.

- Reporte de soplo de intensidad I/VI o II/ VI sin presencia de anemia o con coloración rosada de mucosas.

- Reporte de cuadro clínico compatible con alguna cardiopatía y con radiografía torácica que muestra incremento de la silueta cardiaca o con tráquea desplazada dorsalmente. 
- Reporte de cuadro clínico compatible con alguna cardiopatía y ECG con arritmia patológica.

- Reporte de cuadro clínico compatible con alguna cardiopatía y con ecocardiograma que muestra dilatación cameral cardiaca, engrosamiento de alguna válvula, incremento del índice aurícula izquierda/arteria aorta, o fracción de acortamiento alterada.

Los criterios de exclusión fueron:

- Fichas clínicas sin reporte de cuadro compatible con alguna cardiopatía.

- Reporte de soplo intensidad I/VI o II/VI con examen hematológico con anemia, o que cursaba con alguna enfermedad que podría causar anemia o con hallazgo de mucosas pálidas o color rosa pálido al examen físico.

- Reportes con evidencia de incremento de la silueta cardiaca a la radiografía torácica, pero sin sintomatología de enfermedad cardiaca o ausencia de anomalías al examen físico.

- Reporte sin cuadro clínico característico de cardiopatía con hallazgo de soplo cardiaco al examen físico sin haber especificado la intensidad.

Se determinó la frecuencia de enfermedades cardiacas para el periodo 2007-2009 y por año, tomando en consideración las variables sexo, raza, grupo etario (menos de 1,1 a 4,5 a 8,9 a 12 , más de 12 años), peso (menos de 10,11 a 20, más de $20 \mathrm{~kg}$ ), signos en la historia clínica, y signos clínicos en el examen físico. Para el análisis estadístico se empleó el programa Stata 12 v. 2011. Asimismo, se evaluó el riesgo de tener enfermedades cardiacas con respecto a las variables sexo (machos vs. hembras), edad (rango de menor edad vs. demás rangos de edad) y peso (rango de menor peso vs demás rangos de peso)

\section{Resultados y Discusión}

El número de canes diagnosticados con alguna cardiopatía fue de 260 (2.0\%), correspondiendo a 79 casos en 2007 (1.9\%), 73 en $2008(1.7 \%)$ y 108 en $2009(2.4 \%)$. Estas cifras difieren del $10 \%$ mínimo que se esperaba encontrar en base al reporte del panel de consenso de la especialidad de cardiología del Colegio Americano de Medicina Interna Veterinaria (Tilley et al., 2008), así como otros estudios retrospectivos en Europa, EEUU y Brasil (Eichelberg y Seine, 1996; Hand et al., 2000; Castro et al., 2009), e incluso de la bibliografía latinoamericana sobre enfermedades cardiacas (Belerenian et al., 2001).

No obstante, al evaluar las frecuencias de presentación de cardiopatías por grupo etario, la frecuencia se incrementa a $6.3 \%$ para el grupo de 9 a 12 años y a $15.5 \%$ para el grupo mayor de 12 años (Cuadro 1); es decir, la población geriátrica resultó ser la más afectada. Estas cifras, si bien son menores a las frecuencias referidas por Mucha (2009) ( $25 \%$ en mayores a 9 años y $35 \%$ en mayores a 12 años), coinciden en afirmar que el porcentaje de enfermos cardiacos se incrementa con la edad.

En el análisis de riesgo, teniendo como referencia al grupo etario < 1 año de edad, se determinó que los perros de los grupos etarios de 1 a 4,5 a 8,9 a 12 y $>12$ presentaron un riesgo de presentar problemas cardíacos de 8,28 y 76 veces mayor, respectivamente, que el grupo etario menor de un año (Cuadro 2).

La enfermedad cardiaca con mayor frecuencia de diagnóstico fue la enfermedad crónica de las válvulas auriculoventriculares (ECVAV), que constituyó casi las tres cuartas partes del total de las enfermedades cardiacas (Cuadro 3); sin embargo, no se 
Cuadro 1. Frecuencias de cardiópatas en caninos atendidos en la Clínica de Animales Menores de la Facultad de Medicina Veterinaria, UNMSM, Lima, según grupo etario (periodo 2007-2009)

\begin{tabular}{cccc}
\hline \multirow{2}{*}{$\begin{array}{c}\text { Edad } \\
\text { (en años })\end{array}$} & $\begin{array}{c}\text { Total de fichas clínicas } \\
\text { (n) }\end{array}$ & \multicolumn{2}{c}{ Casos de cardiópatas } \\
\cline { 3 - 4 } & 3,725 & 9 & $\%$ \\
\hline$<1$ & 3,690 & 4 & 0.2 \\
1 a 4 & 2,795 & 53 & 0.1 \\
5 a 8 & 1,737 & 110 & 1.9 \\
9 a 12 & 534 & 83 & 6.3 \\
$>12$ & $12,481^{1}$ & 259 & 15.5 \\
\hline Total & &
\end{tabular}

${ }^{1}$ La edad solo estaba consignada en 12,481 fichas clínicas

Cuadro 2. Valores de Odds Ratio para las variables sexo, peso y edad de perros cardiópatas caninos $(\mathrm{n}=244)$ atendidos en la Clínica de Animales Menores de la Facultad de Medicina Veterinaria, UNMSM, Lima, según el peso corporal (periodo 2007-2009)

\begin{tabular}{lllc}
\hline Variable & & Odds ratio & $\begin{array}{c}\text { Intervalo de confianza } \\
95 \%\end{array}$ \\
\hline Sexo & Macho & 1.3 & $(1-1.7)$ \\
& Hembra & referencia & - \\
Edad (años) & $<1$ & referencia & - \\
& $1-4$ & $0.4^{*}$ & $(0.1-1.5)$ \\
& $5-8$ & $7.9^{*}$ & $(3.9-16.2)$ \\
& $9-12$ & $27.9^{*}$ & $(14.1-55.2)$ \\
Peso $(\mathrm{kg})$ & $>12$ & $75.9^{*}$ & $(37.9-152.2)$ \\
& $<10$ & referencia & - \\
& $11-20$ & 0.7 & $(0.5-1.0)$ \\
& $>20$ & $0.4^{*}$ & $(0.2-0.5)$ \\
\hline
\end{tabular}

${ }^{*} p<0.05$

${ }^{1}$ Intervalo de confianza al $95 \%$ 
pudo establecer el tipo de cardiopatía en el $14.2 \%$ de los casos, debido mayormente a la falta de datos en el examen físico o carencia de exámenes complementarios. Esto, no obstante, indica que las frecuencias reales de cada grupo podrían ser mayores.

En el Cuadro 4 se muestra el detalle de las frecuencias de cada grupo de enfermedad cardiaca por grupo etario, donde se puede observar que la mayor cantidad de casos se encuentra en los dos grupos de mayor edad.

Las cinco razas con mayor frecuencia de diagnóstico de cardiopatías fueron el Pekinés, Shih-Tzu, Cocker, Boxer y el tipo cruzado (Cuadro 5). Los ejemplares de la raza Pekinés fueron los más afectados.

Respecto al sexo, se halló 1.6 machos cardiópatas por cada hembra cardiópata, pero sin diferencia estadística $(\mathrm{OR}=1.3$, Cuadro 2), lo cual contrasta con los hallazgos de otros autores (Morgan et al., 2004; Herrtage, 2006;
Cuadro 3. Frecuencias de tipos de cardiopatías en caninos cardiópatas $(\mathrm{n}=260)$ atendidos en la Clínica de Animales Menores de la Facultad de Medicina Veterinaria, UNMSM, Lima (periodo 2007-2009)

\begin{tabular}{lc}
\hline Cardiopatía & $\begin{array}{c}\text { Frecuencia } \\
(\%)\end{array}$ \\
\hline $\begin{array}{l}\text { Enfermedad crónica de las } \\
\text { válvulas auriculoventriculares }\end{array}$ & 71.9 \\
$\begin{array}{l}\text { Sin establecer cardiopatía } \\
\text { exacta }\end{array}$ & 14.2 \\
Cardiomiopatía dilatada & 5.0 \\
Cardiopatía congénita & 3.9 \\
Arritmia cardiaca & 1.9 \\
Neoplasia cardiaca & 1.9 \\
Cardiopatía hipertrófica & 0.4 \\
canina & \\
Dirofilariasis & 0.4 \\
Efusión pericárdica idiopática & 0.4 \\
\hline Total & 100.0 \\
\hline
\end{tabular}

Cuadro 4. Frecuencias de tipos de cardiopatías en caninos cardiópatas $(n=259)$ atendidos en la Clínica de Animales Menores de la Facultad de Medicina Veterinaria, UNMSM, Lima, según grupo etario (periodo 2007-2009)

\begin{tabular}{|c|c|c|c|c|c|c|}
\hline \multirow{2}{*}{ Cardiopatía } & \multicolumn{5}{|c|}{ Años de edad } & \multirow{2}{*}{$\begin{array}{c}\text { Total } \\
\text { (n) }\end{array}$} \\
\hline & $<1$ & 1 a 4 & 5 a 8 & 9 a 12 & $>12$ & \\
\hline $\begin{array}{l}\text { Enfermedad valvular crónica de las } \\
\text { válvulas auriculoventriculares }\end{array}$ & 0 & 0 & 36 & 84 & 67 & 187 \\
\hline Sin establecer cardiopatía exacta & 0 & 1 & 8 & 13 & 14 & 36 \\
\hline Cardiomiopatía dilatada & 0 & 1 & 4 & 8 & 0 & 13 \\
\hline Cardiopatía congénita & 9 & 1 & 0 & 0 & 0 & 10 \\
\hline Arritmia cardiaca & 0 & 0 & 1 & 3 & 1 & 5 \\
\hline Neoplasia cardiaca & 0 & 1 & 2 & 2 & 0 & 5 \\
\hline Cardiopatía hipertrófica canina & 0 & 0 & 0 & 0 & 1 & 1 \\
\hline Dirofilariasis & 0 & 0 & 1 & 0 & 0 & 1 \\
\hline Efusión pericárdica idiopática & 0 & 0 & 1 & 0 & 0 & 1 \\
\hline Total & 9 & 4 & 53 & 110 & 83 & 259 \\
\hline
\end{tabular}


Cuadro 5. Frecuencias de cardiópatas caninos $(n=260)$ atendidos en la Clínica de Animales Menores de la Facultad de Medicina Veterinaria, UNMSM, Lima, según la raza $^{1}$ (periodo 2007-2009)

\begin{tabular}{lc}
\hline Raza & $\begin{array}{c}\text { Frecuencia } \\
(\%)\end{array}$ \\
\hline Cruzado & 36.3 \\
Pekinés & 18.0 \\
Shih-Tzu & 9.8 \\
Cocker & 6.3 \\
Boxer & 4.3 \\
\hline Total & 74.7 \\
\hline ' Datos de las cinco razas con mayor \\
\multicolumn{2}{c}{ número de casos de cardiopatías }
\end{tabular}

Cuadro 7. Frecuencias de presentación de signos clínicos en canes cardiópatas $(\mathrm{n}=260)$ atendidos en la Clínica de Animales Menores de la Facultad de Medicina Veterinaria, UNMSM, Lima (periodo 2007-2009)

\begin{tabular}{lc}
\hline Signo clínico & $\begin{array}{c}\text { Frecuencia } \\
(\%)\end{array}$ \\
\hline Soplo cardiaco & 87.3 \\
Auscultación pulmonar & 23.9 \\
anormal & 19.3 \\
Caque xia & 13.5 \\
Mucosas pálidas & 13.5 \\
Ascitis & 9.7 \\
Pulso femoral débil & 6.2 \\
Tiempo de llenado capilar & 5.4 \\
retardado & 5.0 \\
Latidos cardiacos irregulares & 4.6 \\
FC/PF ${ }^{1}$ no acordes & 3.5 \\
Edema subcutáneo & 2.3 \\
Mucosas cianóticas & Sonidos cardiacos apagados \\
\hline${ }^{1}$ Frecuencia cardiaca / Pulso femoral
\end{tabular}

Cuadro 6. Frecuencias de cardiópatas caninos $(n=244)$ atendidos en la Clínica de Animales Menores de la Facultad de Medicina Veterinaria, UNMSM, Lima, según el peso corporal (periodo 2007-2009)

\begin{tabular}{lc}
\hline $\begin{array}{l}\text { Peso } \\
(\mathrm{kg})\end{array}$ & $\begin{array}{c}\text { Frecuencia } \\
(\%)\end{array}$ \\
\hline$<10$ & 70.9 \\
11 a 20 & 16.4 \\
$>20$ & 12.7 \\
\hline Total & 100.0 \\
\hline
\end{tabular}

Mucha, 2009), quienes afirman que la frecuencia de enfermedades cardiacas es mayor en los machos.

Respecto al peso corporal, se encontró un incremento muy notorio en la cantidad de cardiópatas a medida que el peso disminuía. Así, el 70.9\% del total de cardiópatas registraron pesos iguales o menores a $10 \mathrm{~kg}$. De esta manera, los perros con peso mayor a 20 $\mathrm{kg}$ tuvieron $60 \%$ menos riesgo que los de peso menor a $10 \mathrm{~kg}$ de presentar problemas cardiacos $(\mathrm{OR}=0.4$, Cuadro 2$)$.

$\mathrm{Al}$ analizar los signos registrados en las historias clínicas de los canes cardiópatas, se encontró que la tos y la disnea fueron los más frecuentes con 45.9 y $31.7 \%$ de ocurrencia. En menor grado se presentó síncope (14.3\%) e intolerancia al ejercicio (12.7\%). Cabe indicar que un paciente pudo haber mostrado más de un signo clínico. La tos cardiogénica se debe a la compresión mecánica del bronquio principal izquierdo por la dilatación de la aurícula izquierda, y también por presencia de edema pulmonar (Tilley et al., 2008).

Dentro del examen físico, el soplo cardiaco fue, de lejos, el de mayor frecuencia, pues fue hallado en el $87.3 \%$ del total de 
pacientes cardiacos (Cuadro 7). Esto se explica dado que la principal enfermedad cardiaca hallada fue de tipo valvular (ECVAV). En estas, el soplo se origina del reflujo de sangre a través de las válvulas cardiacas por una mala coaptación de las mismas (Belerenian et al., 2001).

\section{Conclusiones}

- La frecuencia de cardiopatías en caninos atendidos en la Clínica de Animales Menores de la Facultad de Medicina Veterinaria, UNMSM, en el periodo 20072009 fue de $2.0 \%$ y de $8.5 \%$ cuando se consideraron los canes mayores de ocho años de edad.

- La enfermedad crónica de las válvulas auriculoventriculares fue la cardiopatía más frecuente en caninos, presentándose en el $71.9 \%$ del total de pacientes cardiópatas.

\section{Literatura Citada}

1. Atkins C, Bonagura J, Ettinger $S$, Fox P, Gordon S, Häggström J, Hamlin $\boldsymbol{R}$, et al. 2009. Guidelines for the diagnosis and treatment of canine chronic valvular heart disease. J Vet Intern Med 23: 1142-1150.

2. Belerenian GC, Mucha CJ, Camacho AA. 2001. Afecciones cardiovasculares en pequeños animales. Buenos Aires: Inter-Médica. $360 \mathrm{p}$.

3. Castro MG, Veado JCC, Silva EF, Araújo RB. 2009. Estudo retrospectivo ecodopplercardiográfico das principais cardiopatías diagnosticadas em cães. Arq Bras Med Vet Zootec 61: 1238-1241.

4. Detweiler DK, Patterson DF, Hubben K Botts RP. 1961. The prevalence of spontaneously occurring cardiovascular disease in dogs. Am J Public Health 51: 228-241.

5. Eichelberg H, Seine R. 1996. Life expectancy and cause of death in dogs. I. The situation in mixed breeds and various dog breeds. Berl Munch Tierarztl Wochenschr 109: 292-303.

6. Fioretti M, Delli CE. 1988. Epidemiological survey of dilatative cardiomyopathy in dogs. Veterinaria 2 : 81 (Abstr).

7. Freeman LM, Rush JE. 2006. Enfermedades cardiovasculares: influencia de la nutrición. En: Pibot P, Biourge V, Elliott D (eds). Enciclopedia de la nutrición clínica canina. Gard: Aniwa SAS. p 335-361.

8. Hand MS, Thatcher CD, Remillard RL, Roudebush P, Lewis LD. 2000. Small animal clinical nutrition. $4^{\text {a }}$ ed. Buenos Aires: Intermédica. $1368 \mathrm{p}$.

9. Herrtage ME. 2006. Alteraciones cardiovasculares. En: Schaer M (ed). Medicina clínica del perro y el gato. Madrid: Elsevier. p 121-162.

10. Jourde LM. 2002. Contribution a l'étude des cardiopathies des carnivores domestiques a l'École Nationale Vétérinaire de Toulouse: creation d'une base de données. Tesis doctoral. Toulouse, Francia: Écol. Nation. Vétérinaire de Toulouse. $158 \mathrm{p}$.

11. Morgan RV, Bright RM, Swartout MS. 2004. Clínica de pequeños animales. $4^{\mathrm{a}}$ ed. Madrid: Elsevier. 1355 p.

12. Mucha CJ. 2009. Patologías cardiacas caninas: Incidencia e importancia clínica. Buenos Aires: Portal Cardiología Veterinaria. [Internet], [22 diciembre 2011]. Disponible en: http:// www.cardiologiaveterinaria.com/media/ pdfs/Patologias $\% 20$ cardiacas $\% 2$ 0caninas\% 20inciden-cia\%20y\%20tratamientos.pdf

13. Tilley LP, Smith FW, Oyama MA, Sleeper MM. 2008. Manual of canine and feline cardiology. $4^{\text {th }}$ ed. Missouri: Elsevier. 443 p. 